\title{
PARTIAL HENSELIZATIONS
}

\author{
ROBERT W. SHEETS \\ Department of Mathematics \\ Southeast Missouri State University \\ Cape Girardeau, Missouri 63701 \\ (Received January 28, 1980)
}

ABSTRACT. We define and note some properties of $k$ H-pairs (k Henselian pairs), $\mathrm{k} \mathrm{N}$-pairs, and $\mathrm{kN}^{\prime}$-pairs. It is shown that the 2-Henselization and the 3Henselization of a pair exist. Characterizations of quasi-local $2 \mathrm{H}-$ pairs are given, and an equivalence to the chain conjecture is proved.

KEY WORDS AND PHRASES. $k$ Henselian pair, $k N$-pair, $k N^{\prime}$-pair, chain conjecture. 1980 MATHEMATICS SUBJECT CLASSIFICATION CODES. $13 \mathrm{~J} 15$.

1. INTRODUCTION.

We define a pair ( $\mathrm{A}, \mathrm{m}$ ) to be a $\mathrm{kH}$-pair (a $\mathrm{k}$ Henselian pair) in case the ideal $m$ is contained in the Jacobson radical of the commutative ring $A$ and if for every monic polynomial $\mathrm{f}(\mathrm{X})$ of degree $k$ in $A[X]$ such that $\overline{\mathrm{f}}(\mathrm{X}) \in \mathrm{A} / \mathrm{m}[\mathrm{X}]$ factors into $\bar{f}(X)=\bar{g}_{O}(X) \bar{h}_{O}(X)$ where $\bar{g}_{O}(X)$ and $\bar{h}_{O}(X)$ are monic and coprime, there exist monic polynomials $g(x), h(X) \in A[X]$ such that $f(X)=g(X) h(X), \bar{g}(X)=\bar{g}_{O}(X)$, and $\bar{h}(X)=\bar{h}_{0}(X)$. It is shown that the 2-Henselization and the 3-Henselization of a pair (A,m) exist. Several properties of $\mathrm{k} H$-pairs are noted. And an equivalence to the Chain Conjecture is also given.

2. $\mathrm{k}$ H-PAIRS, $\mathrm{k}$ N-PAIRS, AND $k \mathrm{~N}^{\prime}$-PAIRS.

In this section we define and give some facts about $\mathrm{k} \mathrm{H}$-pairs, $\mathrm{k}$-pairs, and 
k N'-pairs. The main result, Theorem (2.10) states that (i) a $k$ H-pair is a $k$ $\mathrm{N}$-pair, (ii) a k N-pair is a $\mathrm{kN}^{\prime}$-pair, and (iii) an $\mathrm{kN}^{\prime}$-pair is a $\mathrm{f}$ H-pair provided $k \geq \max \left\{c_{j, n} \mid n=0,1, \ldots, j\right\}$.

We begin be stating several definitions. In these definitions and throughout the paper a ring shall mean a commutative ring with an identity element, and $J(A)$ denotes the Jacobson radical of the ring $A$.

DEFINITION 2.1. (A,m) is a pair in case $A$ is a ring and $m$ is an ideal in $A$. DEFINITION 2.2. ( $\mathrm{A}, \mathrm{m})$ is a $\mathrm{k} \mathrm{H}$-pair in case

(i) $\mathrm{m} \subseteq \mathrm{J}(\mathrm{A})$; and

(ii) for every monic polynomial $f(X)$ of degree $k$ in $A[X]$ such that $f(X) \in A / m[X]$ factors into $\bar{f}(X)=\bar{g}_{0}(X) \bar{h}_{0}(X)$ where $\bar{g}_{0}(X)$ and $\bar{h}_{0}(X)$ are monic and coprime, there exist monic polynomials $g(X), h(X) \in A[X]$ such that $f(X)=g(X) h(X)$, $\bar{g}(X)=\bar{g}_{0}(X)$ and $\bar{h}(X)=\bar{h}_{0}(X)$.

DEFINITION 2.3. Let $(A, m)$ be a pair. A monic polynomial $x^{k}+a_{k-1} x^{k-1}+\ldots$ $+a_{1} X+a_{0}$ of degree $k$ is called a $k$ N-polynomial over $(A, m)$ in case $a_{d} \in m$ and $a_{1}$ is a unit mod $m$.

DEFINITION 2.4. (A,m) is a $k$ N-pair in case

(i) $\mathrm{m} \subseteq \mathrm{J}(\mathrm{A})$; and

(ii) every $k \mathrm{~N}$-polynomial over $(\mathrm{A}, \mathrm{m})$ has a root in $\mathrm{m}$.

The next results give some facts about $\mathrm{k} N$-polynomials and $\mathrm{k} N$-pairs.

LEMMA 2.5. Let $\mathrm{f}(\mathrm{X})$ be a $k \mathrm{~N}$-polynomial over the pair $(A, m)$. If $m \subseteq J(A)$, then $f(X)$ has at most one root in $m$.

PROOF. The proof follows from [5, Lemma 1.5], since a $\mathrm{k} \mathrm{N}$-polynomial is an N-polynomial.

REMARK. Every $k \mathrm{~N}$-polynomial over a $\mathrm{k}$-pair $(\mathrm{A}, \mathrm{m})$ has one and only one root in $\mathrm{m}$.

PROPOSITION 2.6. If $(A, m)$ is a $k \mathrm{~N}$-pair, then $(A, m)$ is an $j N$-pair for 2 $2 \leq j \leq k$.

PROOF. Given a $k$ N-pair $(A, m)$, it suffices to show that $(A, m)$ is a $(k-1)$ $\mathrm{N}$-pair. Let $\mathrm{f}(\mathrm{X})$ be a $(\mathrm{k}-1) \mathrm{N}$-polynomial over $(\mathrm{A}, \mathrm{m})$. Let $\mathrm{u}$ be a unit in $\mathrm{A}$ and 
$g(X)=(X+u) f(X)$. Then $g(X)$ is a $k N$-polynomial and thus has a root $r$ in $m$ and $0=g(r)=(r+u) f(r)$. Since $(r+u)$ is a unft, we have $f(r)=0$. Therefore, $(A, m)$ is a $(k-1)$ N-pair.

DEFINITION 2.7. Let $(\mathrm{A}, \mathrm{m})$ be a pair. A monic polynomial $x^{k}+d_{1} x^{k-1}+d_{2} x^{k-2}+\ldots+d_{k}$ of degree $k$ is called a $k N^{\prime}$-polynomial over $(A, m)$ in case $d_{1}$ is a unit mod $m$ and $d_{2}, \ldots, d_{k}$ belong to $m$.

DEFINIPION 2.8. (A,m) is a $k \mathrm{~N}^{\prime}$-pair in case

(i) $\mathrm{m} \subseteq \mathrm{J}(\mathrm{A})$; and

(ii) every $k^{\prime}$-polynomial over $(A, m)$ has a root in $A$, which is a unit. We note that if $(A, m)$ is a $k N^{\prime}$-pair, $f(X)=x^{k}+d_{1} x^{k-1}+\ldots+d_{k}$ is a $k$ $N^{\prime}$-polynomial over $(A, m)$ and $r \in A$ is a root of $f(X)$ given by the definition of a k N'-pair, then $\bar{r}=-\bar{d}_{1}$, and $f^{\prime}(r)$ is a unit.

PROPOSITION 2.9. Let $(A, m)$ be a $k N^{\prime}$-pair, then $(A, m)$ is an $j N^{\prime}$-pair for $2 \leq j \leq k$.

PROOF. Given a $k N^{\prime}$-pair $(A, m)$, it suffices to show that $(A, m)$ is a $(k-1)$ $N^{\prime}$-pair. Let $f(X)$ be a $(k-1) N^{\prime}$-polynomial over $(A, m)$. Then $X f(X)$ is a $k^{\prime}-$ polynomial and has a root $u$, which is a unit. and uf $(u)=0$ implies that $f(u)=0$, therefore $(A, m)$ is a $(k-1) N^{\prime}-p a i r$.

THEOREM 2.10. (i) A kH-pair is a kN-pair

(ii) A kN-pair is a $\mathrm{kN}^{\prime}$-pair

(iii) A kN'-pair is a jH-pair, provided

$k \geq \max \left\{C_{j, n} \mid n=0,1, \ldots, j\right\}$

PROOF. Part (i) follows from the definitions.

The proof of (ii) follows from the proof of [10, Lemma 7$]$

The proof of (iii) follows from Crépeaux's proof of [3, Prop. 1]

\section{3. k N-CLOSURE.}

In this section we construct the $\mathrm{k} N$-closure for a given pair $(\mathrm{A}, \mathrm{m})$. That is, we find the "smallest" k N-pair which "contains" $(A, m)$. The development of this section parallels Greco's development in [5].

In order to construct the $\mathrm{k} \mathrm{N}$-closure we need the following definitions. 
DEFINITION 3.1. A morphism (of pairs) $\emptyset:(A, m) \rightarrow(B, n)$ is a ring homomorphism $\emptyset: \mathrm{A} \rightarrow \mathrm{B}$, such that $\emptyset^{-1}(\mathrm{n})=\mathrm{m}$.

DEFINITION 3.2. A morphism (of pairs) $\emptyset:(A, m) \rightarrow(B, n)$ is strict in case $\mathrm{n}=\emptyset(\mathrm{m}) \mathrm{B}$ and $\emptyset$ induces an isomorphism $\mathrm{A} / \mathrm{m} \rightarrow \mathrm{B} / \mathrm{n}$.

DEFINITION 3.3. Let $(A, m)$ be a pair. A $k$ N-pair $(B, n)$ together with a morphism $\emptyset:(A, m) \rightarrow(B, n)$ is a $\underline{k}$-closure of $(A, m)$ if for any $k N$-pair $\left(B^{\prime}, n^{\prime}\right)$ and any morphism $\Psi:(A, m) \rightarrow\left(B^{\prime}, n^{\prime}\right)$ there exists a unique morphism $\psi^{\prime}:(B, n) \rightarrow\left(B^{\prime}, n^{\prime}\right)$ such that $\Psi^{\prime} \circ \emptyset=\Psi$.

DEFINITION 3.4. Let $(A, m)$ be a pair and $f(X)$ a $k$ N-polynomial over (A,m). Let $A[x]=A[X] /(f(X)), S=1+(m, x) A[x]$ and $B=s^{-1} A[x I$. Then $(B, m B)$ is called a simple $\mathrm{k}$ N-extension of $(A, m)$.

DEFINITION 3.5. A $\underline{\mathrm{k} \text { N-extension }}$ of $(\mathrm{A}, \mathrm{m})$ is a pair obtained from $(\mathrm{A}, \mathrm{m})$ by a finite number of simple $\mathrm{k}$-extensions.

The next two results give some useful properties of simple $k$ N-extensions and k N-extensions.

LEMMA 3.6. Let $(B, n)$ be a simple $k$ N-extension of $(A, m)$. Let $\emptyset: A \rightarrow B$ be the canonical morphism. Then:

(i) $x \in n$.

(ii) $\emptyset^{-1}(\mathrm{n})=\mathrm{m}$ and $\emptyset:(A, m) \rightarrow(B, n)$ is a morphism of pairs.

(iii) $\emptyset:(A, m) \rightarrow(B, n)$ is strict.

PROOF. The proof follows from [5, Lemmas 2.3,2.4, and 2.5] since a simple $\mathrm{k}$ N-extension is a simple $\mathrm{N}$-extension.

COROLLARY 3.7. If $(B, n)$ is a $k$-extension of $(A, m)$, then the canonical morphism $\emptyset:(A, m) \rightarrow(B, n)$ is strict.

We note that a $\mathrm{k}$-extension of a quasi-local ring (A,m) is a quasi-local ring.

The following lemma is used to show that the partial order defined in Definition (3.9) is well defined.

LEMMA 3.8. Let $\left(A^{\prime}, m^{\prime}\right)$ be a $k$ N-extension of $(A, m)$ and let $(B, n)$ be a pair with $n \subseteq J(B)$. Let $\emptyset:(A, m) \rightarrow\left(A^{\prime}, m^{\prime}\right)$ be the canonical morphism. Then for any 
morphism $\Psi:(A, m) \rightarrow(B, n)$ there is at most one morphism $\Psi^{\prime}:\left(A^{\prime}, m^{\prime}\right) \rightarrow(B, n)$ such that $\Psi^{\prime} \circ \emptyset=\Psi$.

PROOF. The proof follows from [5, Lemma 3.1] since a $k$ N-extension is an N-extension.

In particular, the above lemma holds when $(B, n)$ is a $k$-extension of $(A, m)$. DEFINITION 3.9. Define a partial order on the set of $k$-extensions of (A,m) as follows: If $\left(A^{\prime}, m^{\prime}\right)$ and $\left(A^{\prime \prime}, m^{\prime \prime}\right)$ are two $k$-extensions of $(A, m)$, then $\left(A^{\prime}, m^{\prime}\right)$ $\leq\left(A^{\prime \prime}, m^{\prime \prime}\right)$ if and only if there is a morphism $\Psi:\left(A^{\prime}, m^{\prime}\right) \rightarrow\left(A^{\prime \prime}, m^{\prime \prime}\right)$ such that $\Psi \circ \emptyset=\emptyset^{\prime \prime}$, where $\emptyset:(\mathrm{A}, \mathrm{m}) \rightarrow\left(\mathrm{A}^{\prime}, \mathrm{m}^{\prime}\right)$ and $\emptyset^{\prime \prime}:(\mathrm{A}, \mathrm{m}) \rightarrow\left(\mathrm{A}^{\prime \prime}, \mathrm{m}^{\prime \prime}\right)$ are the canonical morphisms.

PROPOSITION 3.10. Let $(A, m)$ be a pair. Then the $k$ N-extensions of $(A, m)$ form a directed set with the order relation and the morphisms defined above.

PROOF. The proof is analogous to $\Gamma 5$, Prop. 3.37 .

LEMMA 3.11 Let $\left(A^{\prime}, m^{\prime}\right)$ be a $k$-extension of $(A, m)$ and let $\emptyset:(A, m) \rightarrow\left(A^{\prime}, m^{\prime}\right)$ be the canonical morphism. Let $(B, n)$ be a $k N-p a i r$ and let $\Psi:(A, m) \rightarrow(B, n)$ be a morphism. Then there is a unique morphism $\Psi^{\prime}:\left(A^{\prime}, m^{\prime}\right) \rightarrow(B, n)$ such that $\Psi=\Psi^{\prime} 0 \emptyset$.

PROOF. The proof is analogous to [5, Prop. 3.4].

THEOREM 3.12. Let $(\mathrm{A}, \mathrm{m})$ be a pair and let $\left(\mathrm{A}^{\mathrm{kN}}, \mathrm{m}^{\mathrm{kN}}\right)$ be the direct limit of the set of all $\mathrm{k}$ N-extensions. Then $\left(\mathrm{A}^{\mathrm{kN}}, \mathrm{m}^{\mathrm{kN}}\right)$ with the canonical morphism $(\mathrm{A}, \mathrm{m}) \rightarrow$ $\left(\mathrm{A}^{\mathrm{kN}}, \mathrm{m}^{\mathrm{kN}}\right)$ is a $\mathrm{k} \mathrm{N}$-closure of $(\mathrm{A}, \mathrm{m})$.

PROOF. The proof is analogous to [5, Thm. 3.5].

We note that if $(A, m)$ is a quasi-local ring; then a $k$ N-closure $\left(A^{k N}, m^{k N}\right)$ of $(A, m)$ is quasi-local, since the direct limit of quasi-local rings is quasi1ocal.

4. $\mathrm{k}$ H-CLOSURES AND AN EQUIVALENCE TO THE CHAIN CONJECTURE.

In this section, we note the existence of a $2 \mathrm{H}-\mathrm{closure}$ and of a $3 \mathrm{H}-\mathrm{closure}$, we give some characterization of a quasi-local $2 \mathrm{H}-$ pair, and we observe that the $\mathrm{H}-\mathrm{closure}$ (or Henselization) of a pair $(\mathrm{A}, \mathrm{m})$ can be written as the direct limit or union of $k$ H-pairs, $k=2,3,4, \ldots$. We also give an equivalence to the Chain Conjecture.

DEFINITION 4.1. Let $(A, m)$ be a pair. A k H-pair $(B, n)$, together with a 
morphism $\emptyset:(A, m) \rightarrow(B, n)$ is a $\underline{k H-c l o s u r e}$ of $(A, m)$ if for any $k H-p a i r ~\left(B^{\prime}, n^{\prime}\right)$ and any morphism $\Psi:(A, m) \rightarrow\left(B^{\prime} n^{\prime}\right)$, there exists a unique morphism $\psi^{\prime}:(B, n) \rightarrow\left(B^{\prime}, n^{\prime}\right)$ such that $\psi^{\prime} \circ \emptyset=\Psi$.

THEOREM 4.2. Let $(\mathrm{A}, \mathrm{m})$ be a pair. Then:

(i) a $2 \mathrm{H}$-closure of $(\mathrm{A}, \mathrm{m})$ is $\left(\mathrm{A}^{2 \mathrm{~N}}, \mathrm{~m}^{2 \mathrm{~N}}\right)$.

(ii) a $3 \mathrm{H}$-closure of $(\mathrm{A}, \mathrm{m})$ is $\left(\mathrm{A}^{3 \mathrm{~N}}, \mathrm{~m}^{3 \mathrm{~N}}\right)$.

PROOF. It suffices to show that a $k N$-closure $(k=2,3)$ is a $k H$-pair. And by theorem 2.10 , we have that a $2 \mathrm{~N}$-pair is a $2 \mathrm{H}$-pair, and that a $3 \mathrm{~N}-\mathrm{pair}$ is a $3 \mathrm{H}-$ pair.

DEFINITION 4.3. If $\emptyset: A \rightarrow B$ is a ring homomorphism, then $B$ is said to be k-integral over $A$ in case each $b \in B$ satisfies a monic polynomial of degree $k$ over $\emptyset(A)$. REMARK. If $B$ is $k$-integral over $A$, then $B$ is also $j$-integral over $A$ for all $\mathbf{j} \geq \mathbf{k}$

In the next three items we give examples of rings and elements which are k-integral over a given ring $A$.

LEMMA 4.4. If $A$ is an integrally closed domain and $f(X) \in A[X]$ is a monic polynomial of degree $k$, then $A[X] /(f(X))$ is $k$-integral over $A$.

PROOF. Let $A[x]=A[X] /(f(X))$ and let $L$ be the quotient field of $A$. Then $[L(x): L] \leq k$ and thus each $\alpha \in A[X]$ satisfies a monic polynomial $g(X) \in L[X]$ of degree $\leq k$. Since $\alpha$ is integral over $A$ and $A$ is integrally closed, it follows that $\mathrm{g}(\mathrm{X}) \in \mathrm{A}[\mathrm{X}]$. Therefore $\mathrm{A}[\mathrm{X}]$ is $\mathrm{k}$-integral over $\mathrm{A}$.

LEMMA 4.5. Let $A$ be a ring and let $f(X)=X^{2}+\alpha X+\beta \in A[X]$. Then $A[X] /(f(X))$ is 2-integral over A.

PROOF. Let $A[x]=A[X] / f(X))$ and then all of the elements of $A[X]$ are of the form $a x+b$ where $a, b \in A$. To show that $A[x]$ is 2-integral over $A$, we need to find $F, G \in$ A such that

$$
(a x+b)^{2}+F(a x+b)+G=0 .
$$

By expanding the left side, we see that $F=a \alpha-2 b$ and $G=a^{2} \beta-b^{2}-F b=a^{2} \beta+b^{2}-a b \alpha$ are the needed values. Therefore $A[X]$ is 2-integral over $A$.

EXAMPLE 4.6. Each element of $\operatorname{End}_{A}\left(A^{k}\right)$ is k-integral over $A$ by [1, Proposition 2.4]. 
In fact, if $M$ is any $A$-module generated by $k$ elements, each element of $\operatorname{End}_{A}(M)$ is k-integral over A.

DEFINITION 4.7. (A,m) is a $(\leq k) H$-pair in case $(A, m)$ is a $f H$-pair for $2 \leq j \leq k$. It follows by Theorem 2.10 that if $(A, m)$ is a $\mathrm{f} \mathrm{N}$-pair (or $\mathrm{f} H$-pair), then $(A, m)$ is a $(\leq k) H$-pair provided $j \geq \max \left\{C_{k, n} \mid n=0,1, \ldots k\right\}$. In particular we have that for $k=2,3$, or 4 , a $k$ H-pair is also a $(\leq k) H$-pair.

LEMMA 4.8. Let $(A, m)$ be a quasi-local domain which is a $(\leq k) H$-pair. Then every $\mathrm{k}$-integral extension domain of $\mathrm{A}$ is quasi-local.

PROOF. The proof is analogus to $[6,(30.5)]$

DEFINITION 4.9. A ring $A$ is decomposed if $A$ is the product of finitely many quasi local rings.

THEOREM 4.10. Let $(A, m)$ be a quasi local ring. Then the following statements are equivalent.

(i) Every finite 2-integral A-algebra $B$ is decomposed.

(ii) Every finite free 2-integral A-algebra B is decomposed.

(iii) Every A-algebra of the form $A[X] /(f(X))$, where $f(X) \in A[X]$ is monic and of degree 2 , is decomposed.

(iv) $(\mathrm{A}, \mathrm{m})$ is a $2 \mathrm{H}$-pair.

PROOF. (i) $\Rightarrow(i i)$ is clear. (ii) $\Rightarrow$ (iii) is clear by (4.5). The proofs that (iii) $\Rightarrow$ (i) and that (iii) $\Leftrightarrow$ (iv) follow classical lines; for example, see [9, Prop. 5, p.2].

THEOREM 4.11. A quasi local domain $(A, m)$ is a $2 \mathrm{H}$-pair if and only if every 2-integral extension domain $A^{\prime}$ of $A$ is quasi-local.

PROOF. $(\Rightarrow)$ is true by $(4.8)$.

$(\Leftrightarrow)$. We will show that $(A, m)$ is a $2 \mathrm{H}$-pair by showing that every finite free 2-integral A-algebra is decomposed. Let $B$ be a finite free 2-integral A-algebra.

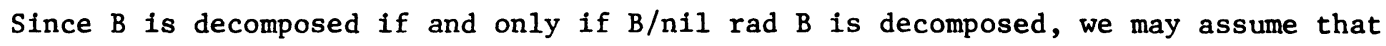
$B$ is reduced. Since $B$ is flat over A, regular elements of A are also regular in B. Thus the minimal primes of $B$ contract to $\{0\}$ in $A$. Let $\left\{P_{i}\right\}_{i} \in I$ be the minimal primes of. $B$. Then for each $\mid i \in I, B / P_{i}$ is a 2-integral extension domain of $A$ and is quasi local by the hypothesis. Thus each minimal prime $P_{i}$ is contained in a unique maximal 
ideal. By [2, Proposition 3, p. 329], the set of minimal primes of B is finite. Let $I_{j}=n_{P_{i}} \subseteq M_{j} P_{i}$ where $M_{j}, j=1, \ldots, n$, are the maximal ideals of $B$. Then the $I_{j}$ are coprime, and $n_{j=1}^{n} I_{j}=0$ since $B$ is reduced. So by the Chinese Remainder Theorem $B \cong \pi_{j=1}^{n} B / I_{j}$ and each $B / I_{j}$ is quasi 1oca1. Thus $B$ is decomposed and therefore $(\mathrm{A}, \mathrm{m})$ is a $2 \mathrm{H}$-pair.

COROLLARY 4.12. Let $(\mathrm{A}, \mathrm{m})$ be a quasi local domain which is $2 \mathrm{H}$-pair. Let $\mathrm{A}^{\prime}$ be an integral extension domain of $A$. If $b \in A^{\prime}$ is 2-integral over $A$, then $b \in J\left(A^{\prime}\right)$ or $b$ is a unit.

PROOF. $A[b]$ is a 2-integral extension domain of $A$ and is thus quasi local. The result follows since all the maximal ideals of $A^{\prime}$ contract to the unique maximal ideal of $\mathrm{A}[\mathrm{b}]$.

We will now show that the $\mathrm{N}$-closure of a pair $(\mathrm{A}, \mathrm{m})$ is the direct limit of the $\mathrm{k} N$-closures of $(\mathrm{A}, \mathrm{m})$. It will follow from this result that the $\mathrm{H}$-closure of $(\mathrm{A}, \mathrm{m})$ can be written as the direct limit of $\mathrm{k}$ H-pairs.

DEFINITION 4.13. Let $(A, m)$ be a pair. Then $(A, m)$ is an $\underline{N-p a i r}$ (respectively, a $\underline{\mathrm{H} \text {-pair) }}$ in case $(\mathrm{A}, \mathrm{m})$ is a $\mathrm{k} \mathrm{N}$-pair (respectively, a $\mathrm{kH}$-pair) for $\mathrm{k}=2,3, \ldots$.

DEFINITION 4.14. Let $(A, m)$ be a pair. An N-pair (respectively, an H-pair) $(B, n)$, together with a morphism $\emptyset:(A, m) \rightarrow(B, n)$ is an $N$-closure (respectively, an $\underline{H-c l o s u r e}$ ) of $(A, m)$ if for any $N$-pair (respectively, any $H$-pair) $\left(B^{\prime}, n^{\prime}\right)$, and any morphism $\Psi: \quad(A, m) \rightarrow\left(B^{\prime}, n^{\prime}\right)$, there exists a unique morphism $\Psi^{\prime}:(B, n) \rightarrow\left(B^{\prime}, n^{\prime}\right)$ such that $\Psi^{\prime} \circ \emptyset=\Psi$.

THEOREM 4.15. Let $(\mathrm{A}, \mathrm{m})$ be a pair. Then the H-closure of $(\mathrm{A}, \mathrm{m})$ is isomorphic to the $\mathrm{N}$-closure.

PROOF. See [5, Lemma 1.4 and Theorem 5.10].

PROPOSITION 4.16. Let $\left(\mathrm{A}^{\mathrm{N}}, \mathrm{m}^{\mathrm{N}}\right)$ be an $\mathrm{N}$-closure of $(\mathrm{A}, \mathrm{m})$. Then $\left(A^{N}, m^{N}\right) \cong \operatorname{dir} \lim \left(A^{k N}, m^{k N}\right)$, where the directed system $\left\{\left(A^{k N}, m^{k N}\right), \mu_{k j}\right\}$ of $k$ $\mathrm{N}$-closures of $(A, m), k=2,3,4, \ldots$, is ordered by $\left(A^{k N}, m^{k N}\right) \leq\left(A^{j N}, m^{j N}\right)$ iff $k \leq j$ and if $k \leq j$, then $\mu_{k j}:\left(A^{k N}, m^{k N}\right) \rightarrow\left(A^{j N}, m^{j N}\right)$ is the unique morphism which makes the following diagram commute: 


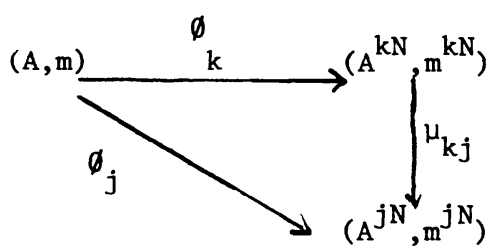

where $\emptyset_{j}$ and $\emptyset_{k}$ are the canonical morphisms.

PROOF. The proof follows immediately from Definitions (3.3) and (4.14) and the definition of a direct limit.

COROLLARY 4.17. Let $\left(\mathrm{A}^{\mathrm{H}}, \mathrm{m}^{\mathrm{H}}\right)$ be the $\mathrm{H}$-closure of $(\mathrm{A}, \mathrm{m})$. Then $\left(\mathrm{A}^{\mathrm{H}}, \mathrm{m}^{\mathrm{H}}\right) \cong$ $\operatorname{dir} \lim \left(A_{i}, M_{i}\right)$ where $\left(A_{i}, m_{i}\right)$ is an $i \underline{H-p a i r}$ for $i=2,3, \ldots$.

PROOF. For a given $i$, let $\left(A_{i}, m_{i}\right)=\left(A^{k N}, m^{k N}\right)$ where $k=\max ^{\prime}\left\{c_{j, n} \mid n=0,1, \ldots, j\right\}$. Then the corollary follows by results $(2.10),(4.15)$ and $(4.16)$.

We now give an equivalence to the Chain Conjecture. The terminology used is the same as in [8] or [10].

THEOREM 4.18. The following statements are equivalent:

(i) The Chain Conjecture holds.

(ii) Every 2 Henselian local domain A, such that the integral closure of A is quasi-local, is catenary.

PROOF. (i) $\Rightarrow$ (ii). This follows by [8, Thm. 2.4].

(ii) $\Rightarrow(1)$. By [8, Thm. 2.4] it suffices to show that every Henselian local domain is catenary. Let $A$ be a Henselian local domain. Then $A$ is also 2 Henselian and the integral closure of $\mathrm{A}$ is quasi-1ocal by $[6,(43.12)]$. Thus by the hypothesis A is catenary.

\section{EXAMPLES.}

In this section we show that there exist $\mathrm{k} \mathrm{N}$-pairs which are not $\mathrm{N}$-pairs and there exist $\mathrm{k} \mathrm{H}$-pairs which are not $\mathrm{H}$-pairs. More precisely, for each prime number $\mathrm{p}$ we give an example of a pair which is not a $\mathrm{p} \mathrm{N}$-pair but is a $\mathrm{k} N$-pair for $2 \leq k<p$. This example also shows that for any integer $k \geq 2$, there exists a $k$ $\mathrm{H}$-pair which is not a $\mathrm{p} \mathrm{H-pair}$ for some sufficiently large prime number $p$.

Let $p>2$ be a prime number. Let $(R, q)$ be a normal quasi-1ocal domain such that there exists an $f(X)=x^{p}+\ldots+a_{1} x+a_{0} \in R[X]$, where $a_{1} \notin q$, $a_{0} \in q$ and $f(x)$ 
is irreducible over $\mathrm{R}[\mathrm{X}]$.

In particular, let $R=Z_{(2)}$ and let $f(x)=X^{P}+3 x+6$. Then by Elsenstein's Criterior, $f(X)$ is irreducible in $Q[X]$, and thus irreducible in $Z_{(2)}[X]$ since $f(X)$ has content 1 .

Let $\mathrm{K}$ be the quotient field of $\mathrm{R}$ and let $\overline{\mathrm{K}}$ be an algebralc closure of $\mathrm{K}$. Let $R^{\prime}$ be the integral closure of $R$ in $\bar{K}$ and $P^{\prime}$ any maximal ideal in $R^{\prime}$. Now $f(X)$ as an element of $R^{\prime}[X]$ factors completely, and since $P^{\prime} \cap / R=q, f(X)$ has a unique root $\alpha \in P^{\prime}$. Let $L$ be the least normal extension of $K$ containing $\alpha$. Then $P \mid[L: K]$ and by [7, Thm. 6] there is a maximal field $M$ without $\alpha$ of exponent $p$ with $\mathrm{K} \subseteq \mathrm{M} \subset \overline{\mathrm{K}}$. Let $\mathrm{A}=\mathrm{R}^{\prime} \cap \mathrm{M}$ and let $\mathrm{m}=\mathrm{P}^{\prime} \Gamma_{1} \mathrm{~A}$.

Now $(A, m)$ is not a $p$ N-pair since $f(X)$ is a $p$-polynomial over $(A, m)$ which does not have a root in $\mathrm{m}$. But $(\mathrm{A}, \mathrm{m})$ is a $\mathrm{k} \mathrm{N}$-pair for $2 \leq \mathrm{k}<\mathrm{p}$. For, let $\mathrm{g}(\mathrm{X})$ be a $(p-1) N$-polynomial over $(A, m)$. Then $g(X)$ as an element of $R^{\prime}[X]$ has a unique root $\beta \in P^{\prime}$. Now $[M(\beta): M] \leq p-1$, but by [7, Thm. 2], $[M(\beta): M]=p^{1}$ for some $i \geq 0$. So $[M(B): M]=1$ and $\beta \in M$. Thus $\beta \in m=P^{\prime} \cap A$ and $(A, m)$ is a $(p-1) N-p a i r$. It follows by (2.6) that $(\mathrm{A}, \mathrm{m})$ is a $\mathrm{kN}$-pair for $2 \leq \mathrm{k}<\mathrm{p}$.

REMARK. If $j$ and the prime number $p$ are closen such that $p>\max \left\{c_{j, n} \mid n=0,1, \ldots, j\right\}$, then by Theorem 2.10, the above example is an example of a pair (A,m) such that $(A, m)$ is not a $p H$-pair, but $(A, m)$ is a $k H$-pair for $2 \leq k \leq j$.

Let the notation be as in the above example. Then $\left(\mathrm{A}_{\mathrm{m}}, \mathrm{mA}_{\mathrm{m}}\right)$ is as an example of a normal quasi-local domain which is not a $\mathrm{p} \mathrm{N}$-pair, but is a $\mathrm{k}$-pair for $2 \leq \mathrm{k}<\mathrm{p}$.

\section{PROPERTIES OF $\mathrm{k}$ N-PAIRS.}

We conclude this paper by noting that many of the properties of the Hensilization or $\mathrm{N}$-closure of a pair which $\mathrm{S}$. Greco proved in [5] also hold for a $\mathrm{k} \mathrm{N}-$ closure and thus also for a $2 \mathrm{H}$-closure and a $3 \mathrm{H}$-closure. Some of these results are: direct limits commute with $\mathrm{k} \mathrm{N}$-closures, cf. [5, Cor. 3.6]; a k N-closure of $(A, m)$ is flat over $A$ and is faithfully flat over $A$ iff $m \subseteq J(A), c f .[5, T h m .6 .5]$; a $\mathrm{k} \mathrm{N}$-closure of a noetherian ring is noetherian, and if a k N-closure of $(\mathrm{A}, \mathrm{m})$ is Noetherian and $\mathrm{m} \subseteq \mathrm{J}(\mathrm{A})$, then $\mathrm{A}$ is Noetherian, cf. [5, Cor. 6.9]; if $\mathrm{A}$ is Noetherian 
and $A$ has one of the properties $R_{k}, S_{k}$, regular, or Cohen-Macaulay, then a $k$ closure of $(\mathrm{A}, \mathrm{m})$ also has that property, and the converse is also true provided $\mathrm{m} \subseteq \mathrm{J}(\mathrm{A})$, cf. [5, Cor. 7.7]; a $\mathrm{k} \mathrm{N}$-closure preserves locally normal, cf. [5, Thm. 9.7]; and $a \mathrm{k}$-closure of a reduced ring is reduced, $\mathrm{cf}$. [5, Thm. 8.7].

\section{REFERENCES}

1. ATIYAH, M.F. and I.G. MACDONALD. Introduction to Commutative Algebra, AddisonWesley Publishing Co., Reading, Mass., 1969.

2. BOURBAKI, NICOLAS. Commutative Algebra, Addison-Wesley Publishing Co., Reading, Mass., 1969.

3. CRÉPEAUX, E. "Une caractérisation des couples Henseliens," L'Enseignement Mathématique 13, (1968), pp. 273-279.

4. GRECO, SILVO. "Algebras over nonlocal Hensel rings," Jour. Algebra 8 (1968), pp. 45-49.

5. GRECO, S. "Henselization of a ring with respect to an ideal," Trans. Amer. Math. Soc. 144 (1969), pp. 43-65.

6. NAGATA, MASAYOSHI. Local Rings, Interscience Publishers, New York, N.Y., 1962.

7. QUIGLEY, FRANK. "Maximal subfields of an algebraically closed field not containing a given element," Proc. Amer. Math. Soc. 13 (1962), pp. 562-566.

8. RATLIFF, L.J., JR. Chain Conjectures and H-Domains, Lecture Notes in Mathematics 311, Springer-Verlag, New York, N.Y., 1973, pp. 222-238.

9. RAYNAUD, MICHEL. Anneaux Locaux Henseliens, Lecture Notes in Mathematics 169, Springer-Verlag, New York, N.Y., 1970.

10. SCHERZLER, EBERHAND. "On Henselian Pairs," Commu. Algebra $\underline{3}$ (1975), PP. 391-404. 


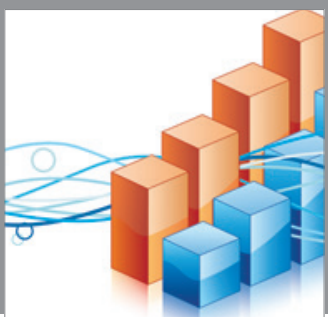

Advances in

Operations Research



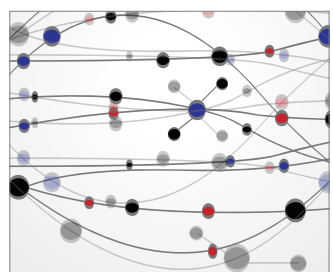

\section{The Scientific} World Journal
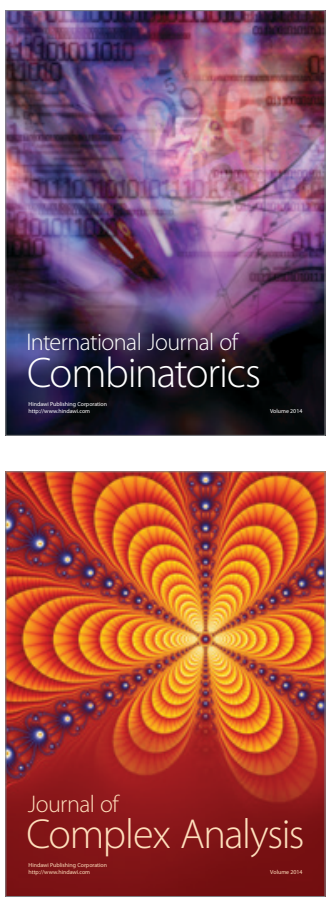

International Journal of

Mathematics and

Mathematical

Sciences
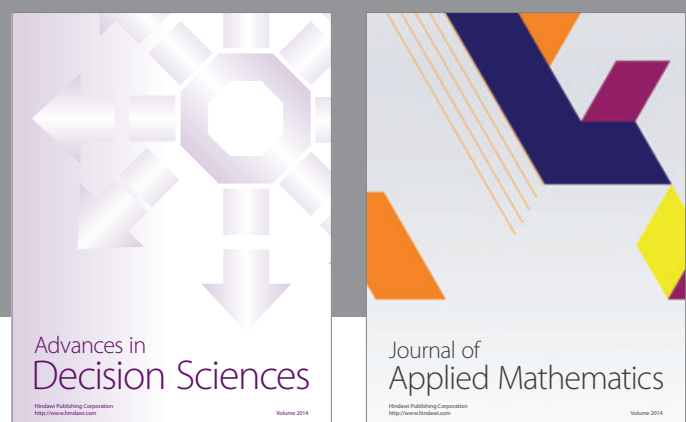

Journal of

Applied Mathematics
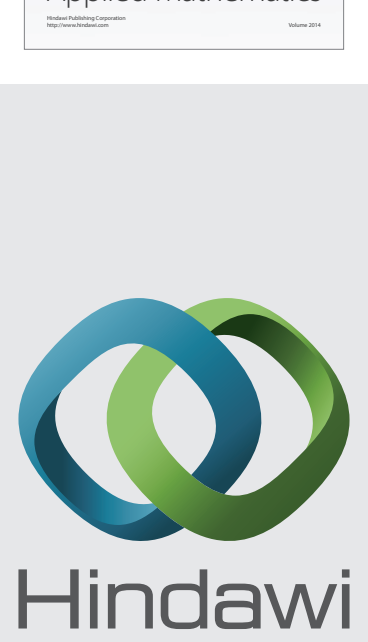

Submit your manuscripts at http://www.hindawi.com
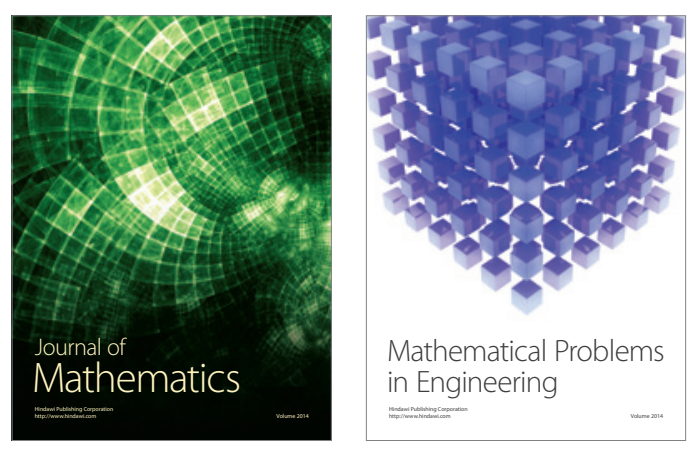

Mathematical Problems in Engineering
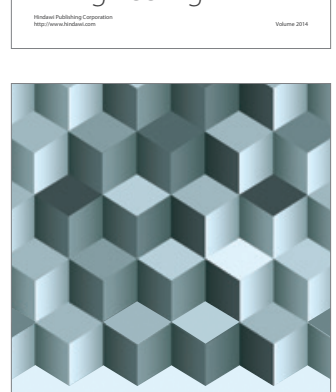

Journal of

Function Spaces
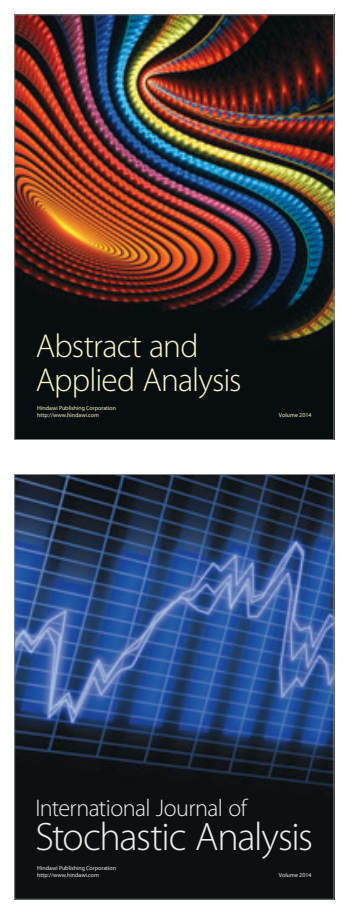

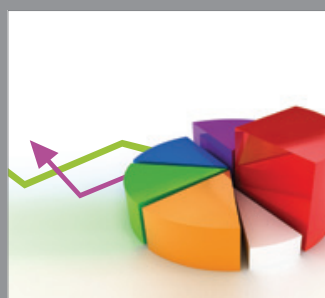

ournal of

Probability and Statistics

Promensencen
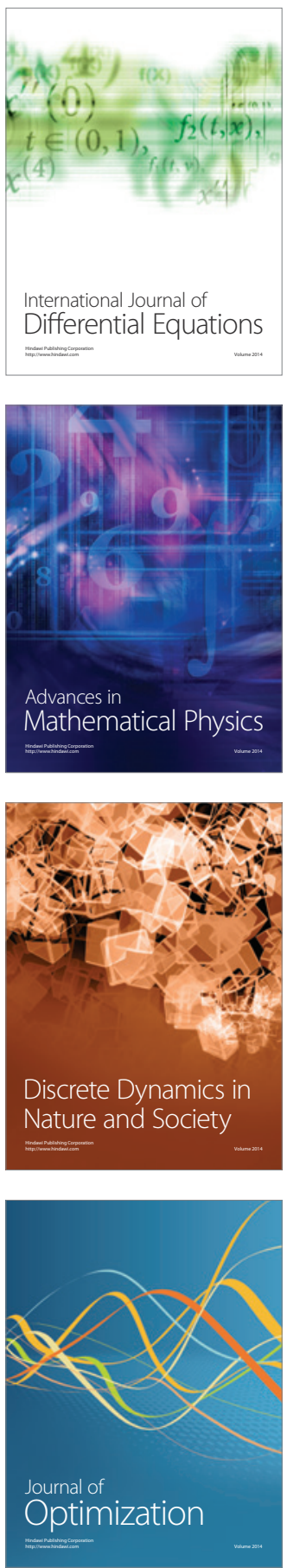